\title{
GCU
}

Glasgow Caledonian

University

University for the Common Good

\section{Optimization of duty cycles for LED based indoor positioning system}

Popoola, Olaoluwa R.; Popoola, Waisu O.; Ramirez-Iniguez, Roberto; Sinanovic, Sinan

Published in:

2016 International Conference for Students on Applied Engineering, ICSAE 2016

DOI:

10.1109/ICSAE.2016.7810219

Publication date:

2017

Document Version

Author accepted manuscript

Link to publication in ResearchOnline

Citation for published version (Harvard):

Popoola, OR, Popoola, WO, Ramirez-Iniguez, R \& Sinanovic, S 2017, Optimization of duty cycles for LED based indoor positioning system. in 2016 International Conference for Students on Applied Engineering, ICSAE 2016. IEEE, pp. 368-372, International Conference for Students on Applied Engineering (ICSAE), 20/10/16. https://doi.org/10.1109/ICSAE.2016.7810219

\section{General rights}

Copyright and moral rights for the publications made accessible in the public portal are retained by the authors and/or other copyright owners and it is a condition of accessing publications that users recognise and abide by the legal requirements associated with these rights.

Take down policy

If you believe that this document breaches copyright please view our takedown policy at https://edshare.gcu.ac.uk/id/eprint/5179 for details of how to contact us. 


\title{
Optimization of Duty Cycles for LED based Indoor Positioning System
}

\author{
Olaoluwa R. Popoola*, Wasiu O. Popoola ${ }^{\dagger}$, Roberto Ramirez-Iniguez* and Sinan Sinanović* \\ ${ }^{*}$ School of Electrical and Built Environment \\ Glasgow Caledonian University, Glasgow, United Kingdom G4 0BA \\ Email: \{olaoluwa.popoola, roberto.ramireziniguez, sinan.sinanovic\}@gcu.ac.uk \\ $\dagger$ Institute for Digital Communications, University of Edinburgh, Edinburgh, EH9 3JL, United Kingdom \\ Email: w.popoola@ed.ac.uk
}

\begin{abstract}
In this paper, an optimal duty cycle, which increases packet delivery ratio (PDR) and reduces probability of collision is designed for packet transmission in an indoor positioning system based on a multiple LED estimation model (MLEM). MLEM improves positioning accuracy by identifying positions of overlap regions where light footprints from multiple LEDs. In MLEM, asynchronous LED transmitters send positional information at the same frequency, amplitude, and wavelength. Consequently, in an LED overlap region, transmitted packets collide and therefore are lost. Methods to avoid packet collision in this region are considered and based on keeping the hardware simple, packet-based pulse duration multiplexing is optimized to provide a time based pseudo-orthogonality between packets. Initial experiments showed a PDR of 0.5 in the overlap region. However, by defining the boundary conditions for non-persistent packet collisions, an exhaustive search for the optimal packet transmission configuration is carried out in this work. Results show that the optimum points occur at 0.09 and 0.1 duty cycles. The use of this optimal duty cycles improved PDR to 0.9 for transmission in the overlap region.
\end{abstract}

Keywords- Indoor localization; optical wireless communications; multiple LEDs; overlap; optimization

\section{INTRODUCTION}

Visible light based data transmission by rapidly switching light emitting diodes (LEDs) involves establishing a communication link between an LED and a photo detector (PD) based receiver. With the recent advances in the design of high switching frequency LEDs, its application in visible light communication (VLC) has increased in importance in research circles [1]. One such application under rigorous study, is the use of VLC-LEDs in the positioning of indoor objects [2]. LED based indoor positioning takes advantage of the high switching capacity of LEDs to transmit positional information to a remote PD-based receiver.

The majority of technologies used in indoor positioning systems (IPS) falls into one of six categories. These are $\mathrm{WiFi}$ and other radio frequency (RF) systems, ultrasound, magnetics, optics, geophones and fingerprinting. These technologies have been applied in the investigation of an optimal strategy for indoor positioning [3]-[5]. However, with the exception of optics or VLC-based indoor positioning, all systems require additional infrastructure for their use. The amount of additional infrastructure required varies based on the technology considered. Whereas WiFi and other RF based localization require multiple additional transmitter sources, ultrasound based systems require a minimum of three additional sound transmitters [4]. Apart from the cost of procuring and installing additional infrastructure, some of these systems broadcast electromagnetic waves which could interfere with the operation of other wireless devices. In data sensitive areas such as a secure military facility or hospitals, the use of these systems would require additional safety measures. VLC-based indoor positioning systems (IPS), however, do not suffer the afore-listed limitations; they do not interfere with RF or WiFi based equipments and LEDs are relatively inexpensive. Since the same LEDs used for lighting is what is used for positioning, VLC-based IPS require no additional infrastructure.

In VLC-based IPS, positional information is sent from an optical source to a mobile optical receiver. This positional information usually carries the location of the LED transmitters and an algorithm is used to decode the position of the mobile receiver based on the positional information of transmitter received. A number of algorithms have been proposed to decode the receiver location from the information [6]-[12]. In [6], a multiple LED estimation model (MLEM) is proposed as a viable method for VLC-based IPS. Unlike other proposed algorithms, MLEM IPS is inexpensive and does not require complex hardware. In MLEM, based on its relatively simple positioning algorithm, packets carrying positional information are susceptible to collision. When collision occurs, the data carrying packets are destroyed and the receiver waits for the next transmission. The amount of time the receiver waits is dependent on the probability of collision in the overlap region. Thus an MLEM system, although simple, may be slow in updating positional information.

In this paper, the time difference between packets in MLEM are investigated with a view to determining the optimal duty cycles that minimize the probability of collision while maintaining or improving the packet delivery ratio. The rest of the paper is organized as follows: Section II establishes the basic system used for simulation in this work and in Section III collision prevention strategies are presented. Section IV] explains the procedure for determination of optimal duty cycle parameters for reduced packet collision. Findings 
from investigations are given in Section $\nabla$ and finally in Section VI the conclusions are presented.

\section{System Model}

Considering a typical room size $5 \mathrm{~m} \times 5 \mathrm{~m} \times 3 \mathrm{~m}$, where four LED transmitters are uniformly distributed at a height $h=2.5 \mathrm{~m}$ from the floor. The power received at any random location in the room is given by $P_{r}=H(0) P_{t}$ where $H(0)$ is the DC channel gain for directed line of sight (LOS) given by [13]-[15]

$H(0)= \begin{cases}\frac{m+1}{2 \pi d^{2}} A \cos ^{m}(\phi) T_{s}(\varphi) g(\varphi) \cos (\varphi), & \text { for } 0 \leq \varphi \leq \varphi_{c} \\ 0, & \varphi>\varphi_{c}\end{cases}$

where $A$ is the physical area of the detector in a PD, $d$ is the LOS distance between the transmitter and the receiver, $\phi$ is the angle of irradiance with respect to the transmitter perpendicular axis and $\varphi$ is the angle of incidence. $T_{s}(\varphi)$ is the gain of an optical filter, $\varphi_{c}$ is the field of vision of the receiver, $g(\varphi)$ is the gain of the optical concentrator given as a proportion of the refractive index $n$ as

$$
g(\varphi)= \begin{cases}\frac{n^{2}}{\sin ^{2} \varphi_{c}}, & 0 \leq \varphi \leq \varphi_{c} \\ 0, & \varphi>\varphi_{c}\end{cases}
$$

and $m$ is the order of the Lambertian source and is given by the semi-angle at half power $\Phi_{(1 / 2)}$ of an LED as

$$
m=\frac{\ln (1 / 2)}{\ln \left(\cos \left(\Phi_{1 / 2}\right)\right)} .
$$

Based on the equations, the simulation parameters shown in Table I and assuming unity gain for the optical filter, the optical received power profile is illustrated in Fig. 1

TABLE I

PARAMETERS FOR SIMULATION

\begin{tabular}{ll}
\hline \multicolumn{2}{c}{ Parameters } \\
\hline Light emitting diode (LED) & \\
semi-angle of half power $\Phi_{1 / 2}$ & $\pm 35^{\circ}$ \\
peak wavelength $\lambda_{p}$ & $860 \mathrm{~nm}$ \\
total radiant flux $\phi_{a}$ & $70 \mathrm{~mW}$ \\
rise and fall time $t_{r}, t_{f}$ & $12 \mathrm{~ns}$ \\
Photodetector (PD) & \\
Directivity $(\theta)$ & $45^{\circ}$ \\
Peak wavelength $\lambda_{p}$ & $950 \mathrm{~nm}$ \\
Minimum irradiance $E_{(\mathrm{emin})}$ & $0.12 \mathrm{~mW} / \mathrm{m}^{2}$ \\
Detector physical area & $1 \mathrm{~cm}^{2}$ \\
Refractive index $n$ & 1.5 \\
Field of Vision $\varphi_{c}$ & $60^{\circ}$ \\
\hline
\end{tabular}

Based on these figures, the four LED transmitters produced no overlaps, two overlaps and four overlaps. Non-overlapping and two-overlap regions account of about $96 \%$ of the room area. In the non-overlapping region, no collision occurs. However, in the two-overlap region, light carrying positional information from one LED is subject to interference with optical signals from another LED. This interference leads to packet collision of the optical transmitted signal. Possible interference handling methods for this scenario are given in Section III

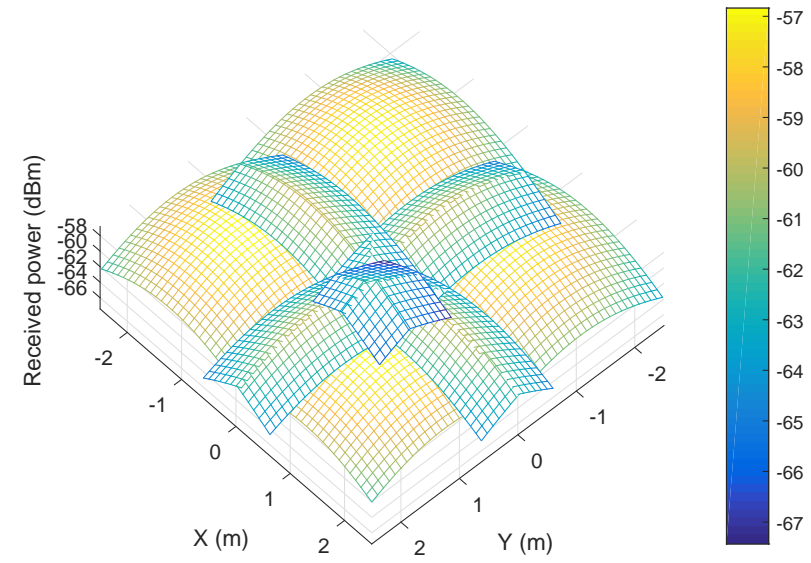

Fig. 1. Bottom view showing distribution of received power with various regions of overlap to be considered.

\section{COLLISION PREVENTION METHODS}

Wireless communication channels, depending on their nature cause data to suffer from one or more channel impairments including noise, attenuation, distortion and interference [16]. The design of MLEM receivers addresses the first three problems and allows for successful data reception in the non-overlapping region. In the overlapping region (Fig. 1) however, interference causes packet destruction. To avoid this interference, signal modulation has been used to separate the signals in frequency, amplitude or phase [16]. More efficient communication techniques use orthogonal codes to prevent interference. However, for MLEM, the receiver system is designed to be simple and inexpensive. MLEM therefore uses the on-off keying (OOK) modulation technique. In MLEM, positional information from multiple transmitters is processed by a single receiver. Thus, the transmitters are designed to be similar in every aspect and differ only in the codes they transmit. A method of interference handling using code multiplexing involves the generation of orthogonal codes. The optical orthogonal codes (OOC) investigated in [17], [18] could be applicable to MLEM. With regards to OOCs, consider two sets of OOCs $p$ and $q$ from [18] with equal auto and crosscorrelation $\lambda_{a}=\lambda_{c}=1$ shown in Fig. 2 a and b. $\lambda_{a}$ represents the auto-correlation and $\lambda_{c}$ is the cross correlation of the two OOCs. Fig. 2k, orthogonal code set $w$, illustrates both sets $p$ and $q$ in the overlap region. The cross correlation condition 
for orthogonality between two codes $p$ and $q$ is given by

$$
\left|Z_{p, q}(l)\right|=\left|\sum_{n=0}^{F-1} p_{n} q_{n+l}\right| \leq \lambda_{c} \quad \text { for } 0 \leq l \leq F-1 .
$$

where $F$ represents the length of $p$ and $q$ and $l$ is an integer that takes any value between $(0$ and $F-1)$. A plot of the cross correlation of codes $p$ and $q$, shown in Fig. 3 indicates that these codes satisfy the condition of orthogonality. However, Fig. 3b illustrates that the cross correlation of OOC $p$ with the code in the overlap $w$ does not satisfy the required condition for orthogonality as given in (4). The MLEM receiver front end frame synchronization requirement does not allow for recognition of this code (OOC set $\mathrm{w}$ ). A packet-based pulse duration multiplexing protocol was therefore proposed in [6] to provide a time based pseudo-orthogonality between packets. This method reduces the probability of collision of packets by transmitting at a low duty cycle but this system is susceptible to multiple repeated collisions.

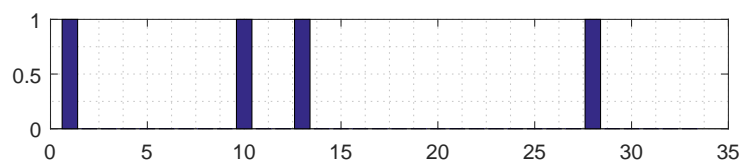

(a) OOC code set $p$

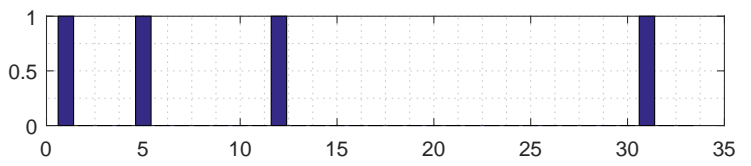

(b) OOC code set $q$

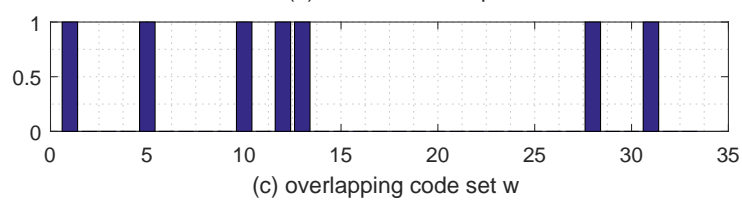

Fig. 2. Distribution of received power for 4 LEDs
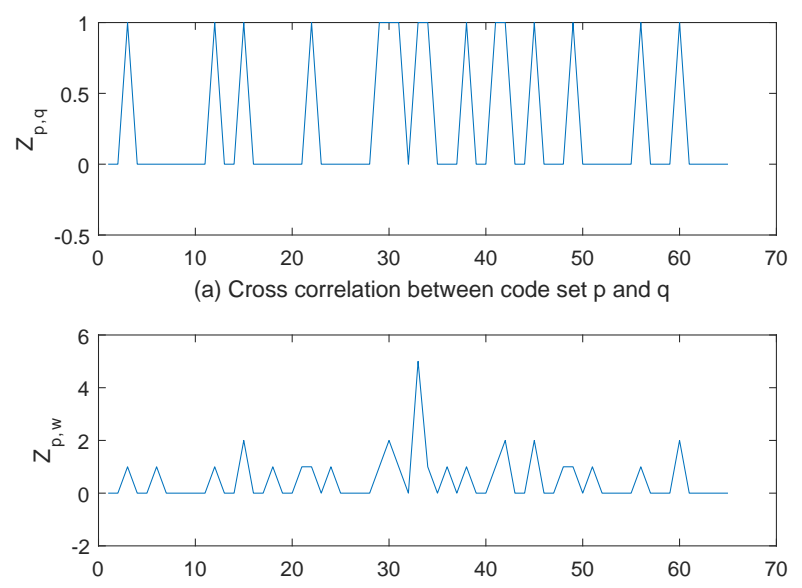

(b) Cross correlation between code set $\mathrm{p}$ and overlapping code set $\mathrm{w}$

Fig. 3. Illustration of cross correlation between codes in the non-overlapping and two-overlap regions

\section{Pulse DURATION MUlTiPleXiNG PROTOCOL OPTIMIZATION}

In this section, the existing pulse duration multiplexing protocol is improved upon by finding optimal duty cycles that reduce the possibility of repeated collision and increase the packet delivery ratio. The duty cycle is dependent on the packet duration and the delay between successive packets. The packet duration is determined by the LED identity code and the encoding scheme applied to the identity code to be transmitted. Existing protocols are encoded by pulse width modulation (PWM), pulse position modulation (PPM), pulse duration modulation (PDM) or biphase (Manchester) coding (BPC). PWM is used in this research due to its short packet duration which is between 25 and $30 \mathrm{~ms}$ depending on the number of ones and zeros in each unique 12-bit LED identity code. For known LED identity codes, the packet duration is constant. The delay between successful packets from different LEDs give variable parameters that are optimized to reduce the probability of collision or increase the packet delivery ratio. The currently existing MLEM system has a packet delivery time and probability of collision relationship shown in Fig. 4. Depending on the reliability of positioning required, two or more packets need to be delivered successfully in order for the system to identify a position. At a duty cycle of 0.1 , positioning decoding will therefore take about $2 \mathrm{~s}$ or more. However, this low duty cycle, with a probability of collision 0.2 , implies 1 in 5 packets are susceptible to collision.

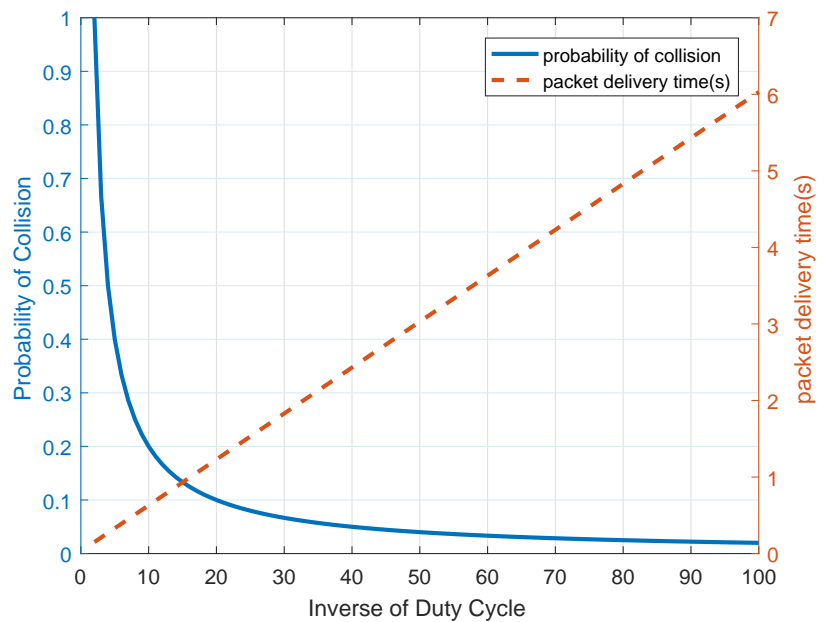

Fig. 4. Packet delivery time and probability of collision vs inverse of duty cycle for two overlapping LEDs

Consider a two-overlap region (Fig. 1), and let the encoded packet frame time be $t_{f 1}$ and $t_{f 2}$, the delay between packets for a first LED be $t_{d 1}$ and the delay between packets for a second LED be $t_{d 2}$ as illustrated in Fig. 5. The conditions for non-persistent collisions are:

$$
\begin{gathered}
0<t_{f 1}<\left|t_{d 1}-t_{d 2}\right| \\
0<t_{f 2}<\left|t_{d 1}-t_{d 2}\right| \\
t_{d 1}>t_{f 1}+t_{f 2} \\
t_{d 2} \geq t_{d 1}+t_{f 1}
\end{gathered}
$$




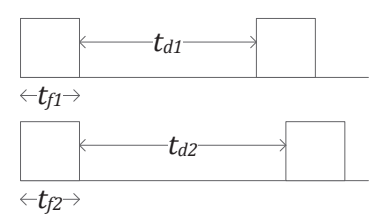

Fig. 5. illustration of time parameters in a two-overlap region

Based on the experimental values for $t_{f 1}$ and $t_{f 2}$, and using the above constraints, we carried out an exhaustive search for the minimum $t_{d 1}$ and $t_{d 2}$ parameters that maximises the packet delivery ratio (PDR) given by:

$$
P D R=\frac{P k_{r}}{P k_{s}}
$$

where $P k_{r}$ is the number of packets received correctly and $P k_{s}$ is the number of packets sent.

A trivial solution is to increase the delay between packets of one of the LEDs infinitely. This will allow for no-collision transmission with a very high PDR from the other LED. This condition however, does not provide sufficient information for positioning in the overlap region (the positional ID from both LEDs are required). Therefore, the search is aimed at finding minimum packet delay times that not only maximise PDR but also ensure that equal PDR is guaranteed for packets from both LEDs. In addition, this optimal points guarantee that the system is not susceptible to repeated collision.

\section{RESULTS}

\section{A. Validation of probability of collision curve}

LED data was generated, encoded with PWM and then modulated using OOK for two LEDs. This data is subsequently subjected to overlapping conditions. Cross correlation of the output with the original encoded LED data informs of the degree of corruption in the received packets. The corrupted packets are discarded and non corrupted packet preserved. This process is repeated for every duty cycle and the probability of collision, taken as the ratio of collided (or destroyed) packets to total number of packets sent is recorded. Fig. 6 shows the results from the simulation of probability of collision plotted with the results of the theoretical expression for probability of collision between packets in the region of overlap between two LEDs by comparison with the simulated probabilities.

\section{B. Optimal duty cycle}

A query to determine of maximum and equal PDRs form Fig. 7 7yielded optimal points for $t_{d 1}$ and $\left|t_{d 1}-t_{d 2}\right|$. From these we calculate the value of $t_{d 1}$ and $t_{d 2}$ at the optimal point called $t_{d 1 o}$ and $t_{d 2 o}$ respectively. Since $t_{f 1}$ and $t_{f 2}$ are constant, $t_{d 1 o}$ and $t_{d 2 o}$ informs the optimal duty cycle for reduced collisions and maximum PDR.

Based on the PWM encoding used, the experimental values for $t_{f 1}$ and $t_{f 2}$ averaged $25 \mathrm{~ms}$. The minimal $t_{d 1}$ and $\left|t_{d 1}-t_{d 2}\right|$ where PDR from the first LED $\left(P D R_{1}\right)$ and PDR from the second LED $\left(P D R_{2}\right)$ are equal and maximum are found to be $226 \mathrm{~ms}$ and $25 \mathrm{~ms}$ respectively. These figures imply a duty cycle of 0.1 and 0.09 respectively. The optimal points are used to run a simulated behaviour of packets from two LEDs shown in Fig. 8 and Fig. $8 \mathrm{~b}$. Fig. $8 \mathrm{~b}$ shows the packets in the overlap region and how the optimal duty cycle reduced collsion.

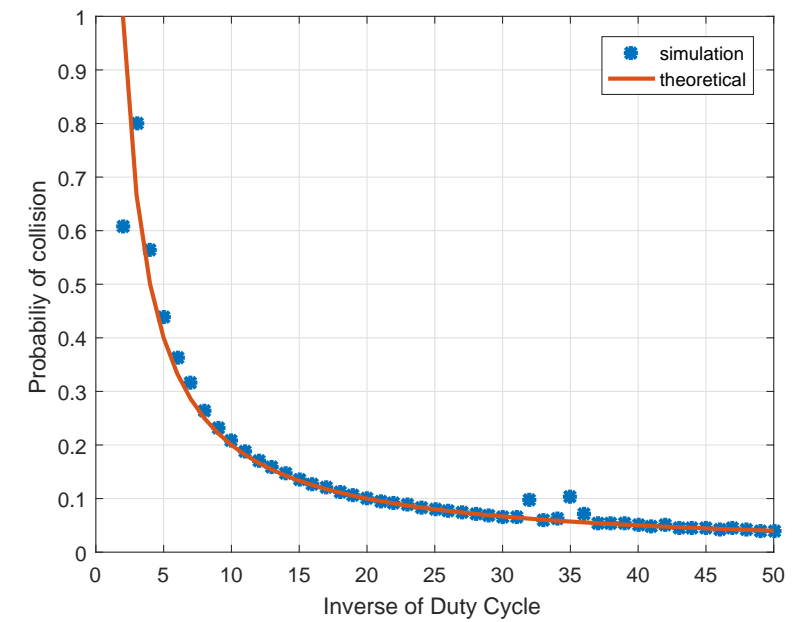

Fig. 6. Theoretical and simulation-based probability of collision vs duty cycle
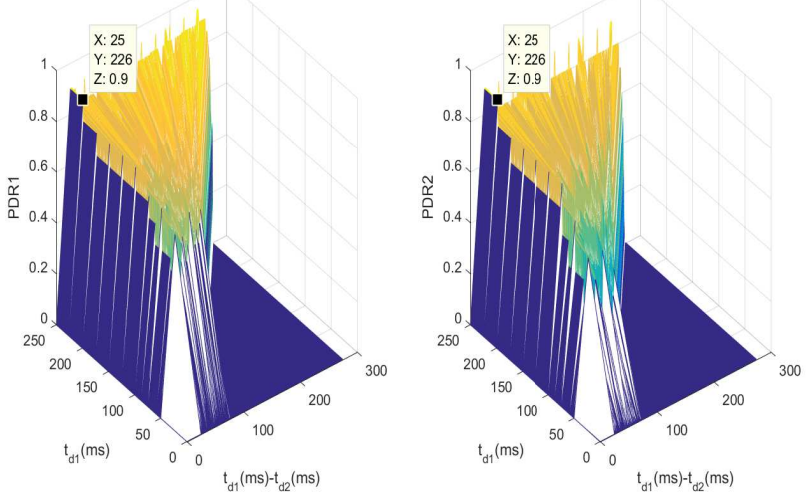

Fig. 7. Output of exhaustive search for optimal PDR

By simulating an MLEM algorithm, recovered packets are illustrated in Fig. $8 \mathrm{~d}$ and Fig. 8 e. In Fig. 8 based on the optimal conditions, a PDR of 0.9 is achieved for both LEDs. This optimized duty cycle condition for packet transfer reduces the probability of packet collision from 0.2 reported in [6] to 0.1 . This system also guarantees that in the occurrence of collision, the next 9 packets from both transmitters are received without collision.

\section{CONCLUSION}

In this paper, the probability of collision for an MLEM system was reduced from 0.2 to 0.1 by finding optimal duty cycles for LED transmission. This reduction corresponds to a packet delivery ratio improvement from 0.5 to 0.9 . Therefore unlike previous designs where only $50 \%$ of packets are guaranteed reception without collision, by designing optimal duty cycles, $90 \%$ of packets are guaranteed reception without collision. The optimal points were found by defining conditions for non-successive collisions. An exhaustive search on all duty cycle configuration possibilities was used to determine the maximum PDR with minimum duty cycles. In future study, the optimum transmission packet configuration is applied to the experimental setup and the effect of improved PDR on 


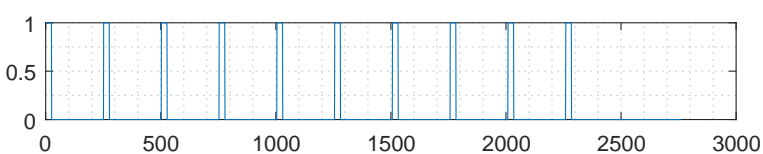

(a) Optimized duty cycle packets for LED L

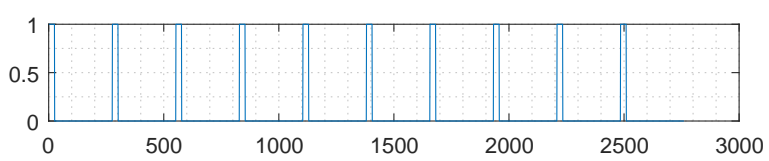

(b) Optimized duty cycle packets for LED $\mathrm{L}_{2}$

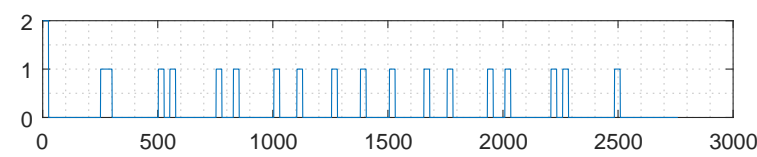

(c) Reduced collision in overlap region

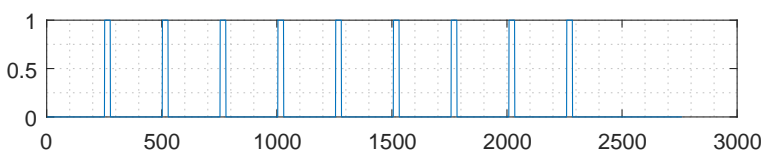

(d) Non-corrupted packets received from $\mathrm{L}_{1}$

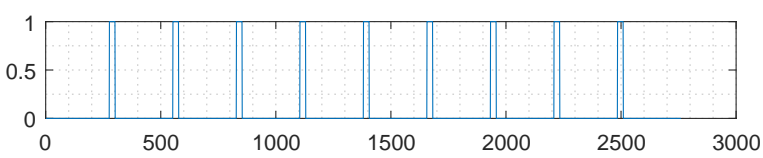

(e) Non-corrupted packets received from $\mathrm{L}_{2}$

Fig. 8. Effect of optimized duty cycle on data-carrying packets

the time taken for the receiver to define a position will be evaluated. In addition the case for regions of four overlaps would be considered.

\section{ACKNOWLEDGMENT}

The authors would like to appreciate the support for this research work from the School of Engineering and Built Environment of Glasgow Caledonian University through the University sponsored research studentship.

\section{REFERENCES}

[1] M. A. Esmail and H. A. Fathallah, "Indoor visible light communication without line of sight: investigation and performance analysis," Photonic Network Communications, vol. 30, no. 2, pp. 159-166, 2015.
[2] J. Lim, "Ubiquitous 3D positioning systems by LED-based visible light communications," IEEE Wireless Communications, vol. 22, no. 2, pp. 80-85, 2015.

[3] Y. Zheng, O. Baala, and A. Caminada, "Efficient design of indoor positioning systems based on optimization model," in Wireless Days (WD), 2010 IFIP. IEEE, 2010, pp. 1-5.

[4] M. Hazas and A. Hopper, "Broadband ultrasonic location systems for improved indoor positioning," IEEE Transactions on mobile Computing, vol. 5, no. 5, pp. 536-547, 2006.

[5] Y. Gu, A. Lo, and I. Niemegeers, "A survey of indoor positioning systems for wireless personal networks," IEEE Communications surveys \& tutorials, vol. 11, no. 1, pp. 13-32, 2009.

[6] O. R. Popoola, F. B. Ogunkoya, W. O. Popoola, R. Ramimez-Iniguez, and S. Sinanovic, "Indoor localization based on multiple LEDs position estimation," IEEE International Workshop on Signal Processing Advances in Wireless Communications, 2016.

[7] A. Papapostolou and H. Chaouchi, "Scene analysis indoor positioning enhancements," annals of telecommunications-annales des télécommunications, vol. 66, no. 9-10, pp. 519-533, 2011.

[8] C. Medina, M. Zambrano, and K. Navarro, "LED based visible light communication: Technology, applications and challenges-a survey," International Journal of Advances in Engineering \& Technology, vol. 8, no. 4, p. 482, 2015.

[9] A. M. Street, P. N. Stavrinou, D. C. O'brien, and D. J. Edwards, "Indoor optical wireless systems-a review," Optical and Quantum Electronics, vol. 29, no. 3, pp. 349-378, 1997.

[10] M. A. Elkarim, N. A. Mohammed, and M. H. Aly, "Exploring the performance of indoor localization systems based on VLC-RSSI, including the effect of NLOS components using two light-emitting diode lighting systems," Optical Engineering, vol. 54, no. 10, pp. 105 110-105 110, 2015.

[11] M. S. Hossen, Y. Park, and K.-D. Kim, "Performance improvement of indoor positioning using light-emitting diodes and an image sensor for light-emitting diode communication," Optical Engineering, vol. 54, no. 4, pp. 045 101-045 101, 2015.

[12] Y. U. Lee and M. Kavehrad, "Two hybrid positioning system design techniques with lighting LEDs and ad-hoc wireless network," IEEE Transactions on Consumer Electronics, vol. 58, no. 4, pp. 1176-1184, 2012.

[13] S.-Y. Jung, S. Hann, and C.-S. Park, "TDOA-based optical wireless indoor localization using LED ceiling lamps," IEEE Transactions on Consumer Electronics, vol. 57, no. 4, pp. 1592-1597, 2011.

[14] W. Gu, W. Zhang, M. Kavehrad, and L. Feng, "Three-dimensional light positioning algorithm with filtering techniques for indoor environments," Optical Engineering, vol. 53, no. 10, pp. 107 107-107 107, 2014.

[15] Z. Ghassemlooy, W. Popoola, and S. Rajbhandari, Optical wireless communications: system and channel modelling with Matlab®. CRC Press, 2012.

[16] J. G. Proakis and M. Salehi, Digital communications. McGraw-Hill international edition, 2008.

[17] F. R. Chung, J. A. Salehi, and V. K. Wei, "Optical orthogonal codes: design, analysis and applications," IEEE Transactions on Information theory, vol. 35, no. 3, pp. 595-604, 1989.

[18] J. A. Salehi, "Code division multiple-access techniques in optical fiber networks. i. fundamental principles," IEEE Transactions on communications, vol. 37, no. 8, pp. 824-833, 1989. 